\title{
The use of closo-dodecaborate-containing linker improves targeting of HNSCC xenografts with radioiodinated chimeric monoclonal antibody U36
}

\author{
JUNPING CHENG ${ }^{1,3}$, MATS ENGSTROM ${ }^{1}$, TOMAS EKBERG ${ }^{1}$, MARIKA NESTOR ${ }^{1,2}$, \\ MATTI ANNIKO $^{1}$ and VLADIMIR TOLMACHEV ${ }^{2}$
}

\author{
${ }^{1}$ Department of Oto-Rhino-Laryngology and Head and Neck Surgery, Uppsala University Hospital (Akademiska Sjukhuset); \\ ${ }^{2}$ Division of Biomedical Radiation, Department of Oncology, Radiology and Clinical Immunology, Uppsala University, \\ Uppsala, Sweden; ${ }^{3}$ Department of Oto-Rhino-Laryngology and Head and Neck Surgery, \\ Tianjin First Central Hospital, Tianjin, P.R. China
}

Received June 17, 2009; Accepted August 19, 2009

DOI: 10.3892/mmr_00000233

\begin{abstract}
Radionuclide imaging of head and neck squamous cell carcinoma (HNSCC) using monoclonal antibodies (MAbs) has the potential to contribute to improved diagnosis and staging, thereby making more effective treatment possible. Chimeric monoclonal antibody U36 (cMAb U36), specific to CD44v6 antigen, is a candidate for the targeting of HNSCC. The aim of this study was to compare the influence of indirect iodination via closo-dodecaborate-based linker (DABI) with the influence of direct radioiodination on the biodistribution of the chimeric anti-CD44v6 antibody U36. The study was performed using nude mice bearing UT-SCC7 HNSCC xenografts using the paired-label method. The biodistribution of cMAb U36 labelled directly with ${ }^{131} \mathrm{I}$ and using DABI with ${ }^{125} \mathrm{I}$ was compared in the same animals. The influence of DABI on the tumour-to-organ ratio was evaluated. For both conjugates, radioactivity uptake in blood and organs decreased with time, except in tumours and the thyroid. DABI-labelled cMAb U36 was characterised by fast blood clearance and an elevated uptake in the liver and spleen. The use of DABI enabled a 1.5 to 2 -fold improvement in the tumour-to-blood and tumour-to-organ ratios in comparison with direct radioiodination, with the exception of the liver and spleen. These results indicate that DABI is a promising linker for the coupling of radioiodine to HNSCC-targeting antibodies.
\end{abstract}

\section{Introduction}

Squamous cell carcinoma, the predominant histological type among cancer of the head and neck (HNSCC), remains the

Correspondence to: Dr Vladimir Tolmachev, Division of Biomedical Radiation, Rudbeck Laboratory, Uppsala University, SE-751 85 Uppsala, Sweden

E-mail: vladimir.tolmachev@bms.uu.se

Key words: nude mice, chimeric monoclonal antibody U36, head and neck squamous cell carcinoma, tumour transplantation, tumour targeting sixth most common neoplasm, accounting for approximately $5 \%$ of all malignant tumours worldwide $(1,2)$. The prognosis for patients with HNSCC has not improved significantly over the past 25 years $(3,4)$. Locoregional recurrence following conventional therapy occurs in approximately $50 \%$ of patients with advanced-stage HNSCC, and distant metastases develop in nearly $25 \%$ of patients $(5,6)$. Treatment with (neo)adjuvant chemotherapy has not led to improvements in the survival of these groups (7). Progress in therapy may be achieved in part by improvements in diagnostics, which would allow for the determination of optimal treatment strategy, and by accurate staging, which would enable the avoidance of over- and undertreatment $(8,9)$.

The introduction of new diagnostic radionuclide-based modalities, such as FDG-PET, appears to be promising for improving the accuracy of staging (10). From a clinical point of view, improved imaging of HNSCC in patients is important for pre-operative evaluation. Nonetheless, a decisive breakthrough in comparison with other diagnostic methods, such as ultrasound, CT or MRI, has yet to be achieved $(11,12)$. In order to improve staging, the radioimmunoscintigraphy of lymph node metastases has been proposed (13). The authors concluded that radioimmunoscintigraphy was not superior to CT or MRI for the detection of lymph node metastases due to its lack of sensitivity; the use of radioimmuno-PET was suggested as a method for improving detection (13).

Iodine-124 $\left(\mathrm{T}_{1 / 2}=100 \mathrm{~h}\right)$ is among the long-lived positron emitters that have a half-life long enough to be compatible with the slow blood clearance of monoclonal antibodies (MAbs) $(14,15)$. The use of PET may increase the sensitivity of radioimmunodiagnostics due to the improved resolution and registration efficiency of this detection technique (16). Recently, direct labelling of an anti-HNSCC cMAb U36 with ${ }^{124}$ I and the characterisation of radioimmunoconjugate was reported (17).

Radiohalogens such as ${ }^{124} \mathrm{I}$ and ${ }^{131} \mathrm{I}$ have certain limitations as antibody labels when conventional labelling methods are used. For example, after binding to the surface of a tumour cell, an antibody is usually internalised and degraded in lysosomes. The leakage of radiocatabolites after degradation results in 
poor intracellular retention (18). This leads to a decrease in tumour-associated radioactivity and the tumour-to-organ radioactivity concentration ratio. Since imaging contrast is proportional to the tumour-to-organ ratio, the sensitivity of tumour detection is negatively affected.

The use of radioiodine linkers charged at lysosomal $\mathrm{pH}$ has the potential to improve the cellular retention of radiocatabolites $(18,19)$. One such linker is negatively charged DABI, which has previously been proposed $(20,21)$. Labelling of the cMAb U36 using DABI did not decrease its affinity for binding to the CD44v6-expressing cell lines SCC25 and SCC9 in comparison with other radioiodination methods (21). The ability of DABI-labelled cMAb U36 to target tumours in vivo has been demonstrated (20). However, a pronounced dependence of tumour radioactivity uptake on tumour size in combination with the limited number of experimental animals used has made it difficult to determine whether the use of DABI improves targeting. One of the problems is the selection of the xenograft model. SCC25 HNSCC cell line xenografts have a long doubling time (32 days) and irregular/irreproducible tumour growth in the nude mice.

In order to refine comparative biodistribution experiments, the following improvements were considered in the current study. First, another HNSCC cell line, UT-SCC7, was proposed as a xenograft in an attempt to shorten the growth time of the animal tumour model. Second, a paired-label technique was adopted. Since the $\gamma$-peaks of ${ }^{125} \mathrm{I}$ and ${ }^{131} \mathrm{I}$ can be completely resolved, two different radioiodinated conjugates may be compared in the same animal. The influence of tumour size on antibody uptake was not crucial in this case, since this factor was the same for both conjugates in each individual tumour.

The objectives of this study were a) to evaluate whether the use of the UT-SCC7 cell line provides a convenient xenograft model for studies on HNSCC targeting and $b$ ) to determine by the dual-label approach whether the use of DABI as a linker for radioiodination provides better targeting of HNSCC in comparison with conventional direct radioiodination.

\section{Materials and methods}

Materials. Sodium metabisulphite and organic solvents were purchased from Merck (Darmstadt, Germany). Chloramine-T and sodium metabisulphite were from Sigma Chemical Company (St. Louis, MO, USA). All chemicals were of analytical grade or better. DABI was prepared at our laboratories as previously described (22). cMAb U36 (Centocor BV, Leiden, The Netherlands), an antibody that recognises the CD44v6 epitope on the outer cell surface (23), was used.

Instrumentation. Radioactivity was measured with the automatic 1480 Wizard $^{\mathrm{TM}}$ 3" Gamma Counter (Wallac AY, Turku, Finland) equipped with a 3 -inch $\mathrm{NaI}(\mathrm{Tl})$ well detector. Size-exclusion chromatography was performed on disposable NAP-5 columns (Amersham Pharmacia Biotech AB, Uppsala, Sweden). The radiochemical purity of the labelled antibody was controlled using the ITLC SG strip (Pall Life Sciences, New York, NY, USA) eluted with $70 \%$ acetone in water. The distribution of radioactivity along the ITLC strips was measured using the Cyclone ${ }^{\mathrm{TM}}$ Storage Phosphor System and analyzed with OptiQuant ${ }^{\mathrm{TM}}$ image analysis software.
Cell line. UT-SCC7 cells (derived from squamous cell carcinoma) were cultured in Dulbecco's modified Eagle's medium (DMEM) supplemented with $10 \%$ foetal calf serum, $2 \mathrm{ml}$ L-glutamine and antibiotics (100 IU penicillin and $100 \mathrm{mg} / \mathrm{ml}$ streptomycin) and non-essential amino acids (10 ml). Cells were grown at $37^{\circ} \mathrm{C}$ in an atmosphere containing humidified air with $5 \% \mathrm{CO}_{2}$.

Animal tumour xenograft model. The study was executed with appropriate licences from the Local Ethics Committee for Animal Research (Project No. C157/2). Animal experiments were performed according to the principles of laboratory animal care. Adult female mice (Balb/c nu/nu; Harlan CPB, Zeist, The Netherlands) were 10 weeks of age at the time of the experiments. Subcutaneous transplantation was performed by injection of $10^{7}$ UT-SCC7 cells into the left flank of the mice. Animals were weighed and tumours were measured every other day for the first 10 days, then the tumours were measured once a day in 3 dimensions with a caliper. Tumour volume was calculated using the ellipsoid formula (volume $=\pi / 6 \mathrm{x}$ length $\mathrm{x}$ width $x$ height). Doubling time was determined after fitting an exponential equation $\mathrm{V}=\mathrm{k}_{1} \mathrm{e}^{\mathrm{k} 2 / \mathrm{t}}$ to the dynamic tumour volume data. From the fitted curve, the doubling time TD $=\ln 2\left(t_{2}-t_{1}\right) /$ $\left(\ln \mathrm{V}_{2}-\ln \mathrm{V}_{1}\right.$ ) was calculated, where $\mathrm{V}_{2}$ and $\mathrm{V}_{1}$ are volumes at $\mathrm{t}_{2}$ and $\mathrm{t}_{1}$, respectively.

Biodistribution experiment and data treatment. cMAb U36 was radioiodinated directly with ${ }^{131} \mathrm{I}$ (conjugate designated as ${ }^{131}$ I-cMAb U36) and with ${ }^{125}$ I using DABI (conjugate designated as ${ }^{125}$ I-DABI-cMAb U36) as previously described $(21,24)$.

Twenty eight days after tumour cell injection, each mouse was injected in the tail vein with a mixture of ${ }^{125} \mathrm{I}-\mathrm{DABI}-\mathrm{cMAb}$ U36 $(60 \mathrm{kBq})$ and ${ }^{131} \mathrm{I}-\mathrm{cMAb} \mathrm{U} 36(40 \mathrm{kBq})$ diluted in PBS. At pre-determined time points, 3, 7, 24, 96 and $192 \mathrm{~h}$ after injection, mice were anaesthetised and sacrificed by heart puncture. Blood was collected and tumour (weight 106-398 mg), brain, salivary gland, heart, lung, stomach, colon, spleen, kidney, liver, bladder and tail (injection site) samples, as well as samples of muscle and bone were excised and placed in pre-weighed plastic vials. After weighing the samples, the radioactivity of each radio-iodine isotope was determined.

For the measurement of ${ }^{125} \mathrm{I}$ and ${ }^{131} \mathrm{I}$ radioactivity, whole spectra of each sample were recorded and treated off-line using a PC. Integral counts in channels 4-38 were considered ${ }^{125}$ I radioactivity and in channels $150-200,{ }^{131} \mathrm{I}$ radioactivity. Data were corrected for spillover, background and dead time. Tissue uptake value, the percent of injected dose/g tissue, was calculated as \% ID/g. Tumour-to-organ ratios were calculated as the ratio of radioactivity concentration in tumours compared to that in each normal organ sample. The contrast enhancement factor was calculated for each sample as the tumour-to-organ ratio for ${ }^{125} \mathrm{I}$ divided by the tumour-to-organ ratio for ${ }^{131} \mathrm{I}$ of the same sample.

\section{Results}

Transplantation and tumour growth. The grafting efficiency of the UT-SCC7 cell line was $100 \%$. Over 20 days, the vitality of the 36 mice was preserved, and their mean weight increased by $1.03 \pm 0.91 \mathrm{~g}$. Calculated from the fitted curve, the tumour 
Table I. Biodistribution of ${ }^{131} \mathrm{I}-\mathrm{cMAb}$ U36 in nude mice bearing the UT-SCC7 HNSCC xenografts.

\begin{tabular}{|c|c|c|c|c|c|}
\hline & $3 \mathrm{~h}$ & $7 \mathrm{~h}$ & $24 \mathrm{~h}$ & $96 \mathrm{~h}$ & $192 \mathrm{~h}$ \\
\hline Blood & $22.06 \pm 4.94$ & $16.11 \pm 3.33$ & $9.50 \pm 2.31$ & $4.85 \pm 1.06$ & $1.74 \pm 0.42$ \\
\hline Tumor & $5.70 \pm 1.83$ & $8.39 \pm 3.57$ & $12.23 \pm 5.40$ & $15.94 \pm 8.95$ & $4.50 \pm 1.00$ \\
\hline Brain & $0.51 \pm 0.37$ & $0.62 \pm 0.25$ & $0.29 \pm 0.06$ & $0.16 \pm 0.06$ & $0.06 \pm 0.04$ \\
\hline Sal gland & $9.22 \pm 2.25$ & $8.05 \pm 0.63$ & $3.29 \pm 0.91$ & $2.04 \pm 0.23$ & $0.57 \pm 0.07$ \\
\hline Heart & $6.30 \pm 1.85$ & $4.56 \pm 1.46$ & $2.51 \pm 0.60$ & $1.13 \pm 0.37$ & $0.33 \pm 0.33$ \\
\hline Lung & $12.61 \pm 2.97$ & $7.92 \pm 3.24$ & $4.28 \pm 1.63$ & $2.23 \pm 0.41$ & $0.82 \pm 0.25$ \\
\hline Stomach & $5.53 \pm 2.50$ & $5.32 \pm 1.89$ & $1.86 \pm 0.79$ & $1.14 \pm 0.36$ & $0.48 \pm 0.13$ \\
\hline Colon & $3.35 \pm 0.76$ & $1.88 \pm 0.77$ & $2.22 \pm 1.35$ & $1.12 \pm 0.45$ & $0.37 \pm 0.15$ \\
\hline Spleen & $12.05 \pm 4.35$ & $5.88 \pm 3.78$ & $2.94 \pm 2.09$ & $1.79 \pm 0.61$ & $0.59 \pm 0.36$ \\
\hline Kidney & $8.20 \pm 1.26$ & $6.38 \pm 1.29$ & $4.05 \pm 0.77$ & $1.99 \pm 0.27$ & $0.70 \pm 0.18$ \\
\hline Liver & $6.10 \pm 0.57$ & $4.65 \pm 1.41$ & $1.63 \pm 0.42$ & $1.23 \pm 0.32$ & $0.39 \pm 0.20$ \\
\hline Bladder & $3.38 \pm 2.62$ & $3.97 \pm 3.25$ & $0.98 \pm 0.83$ & $2.62 \pm 1.41$ & $1.26 \pm 0.21$ \\
\hline Muscle & $1.39 \pm 0.91$ & $0.83 \pm 0.39$ & $0.83 \pm 0.58$ & $0.58 \pm 0.24$ & $0.28 \pm 0.21$ \\
\hline Skeleton & $1.81 \pm 0.37$ & $3.01 \pm 0.90$ & $0.51 \pm 0.19$ & $1.05 \pm 0.35$ & $0.44 \pm 0.62$ \\
\hline
\end{tabular}

Data are expressed as the $\% \mathrm{ID} / \mathrm{g}$ and presented as the average from 5 (occasionally 6 ) animals.

Table II. Biodistribution of ${ }^{131}$ I-DABI-cMAb U36 in nude mice bearing the UT-SCC7 HNSCC xenografts.

\begin{tabular}{lccccc}
\hline & $3 \mathrm{~h}$ & $7 \mathrm{~h}$ & $24 \mathrm{~h}$ & $96 \mathrm{~h}$ & $192 \mathrm{~h}$ \\
\hline Blood & $16.37 \pm 5.60$ & $8.91 \pm 3.06$ & $3.49 \pm 0.84$ & $1.90 \pm 0.51$ & $0.69 \pm 0.23$ \\
Tumor & $3.07 \pm 1.51$ & $4.40 \pm 3.01$ & $4.71 \pm 2.33$ & $9.00 \pm 4.89$ & $2.61 \pm 0.42$ \\
Brain & $0.44 \pm 0.18$ & $0.28 \pm 0.15$ & $0.10 \pm 0.04$ & $0.07 \pm 0.01$ & $0.02 \pm 0.01$ \\
Sal gland & $2.27 \pm 1.03$ & $1.60 \pm 0.89$ & $0.90 \pm 0.27$ & $0.60 \pm 0.12$ & $0.19 \pm 0.04$ \\
Heart & $3.21 \pm 1.23$ & $2.19 \pm 0.76$ & $0.76 \pm 0.18$ & $0.43 \pm 0.05$ & $0.15 \pm 0.05$ \\
Lung & $6.87 \pm 2.87$ & $4.36 \pm 2.91$ & $1.52 \pm 0.48$ & $1.21 \pm 0.69$ & $0.40 \pm 0.08$ \\
Stomach & $2.78 \pm 2.13$ & $1.21 \pm 0.66$ & $0.60 \pm 0.22$ & $0.31 \pm 0.07$ & $0.10 \pm 0.03$ \\
Colon & $2.75 \pm 1.32$ & $2.37 \pm 0.99$ & $0.99 \pm 0.59$ & $0.46 \pm 0.12$ & $0.14 \pm 0.04$ \\
Spleen & $53.33 \pm 36.20$ & $20.00 \pm 25.99$ & $5.71 \pm 4.73$ & $4.06 \pm 2.34$ & $1.60 \pm 1.13$ \\
Kidney & $5.51 \pm 1.90$ & $3.82 \pm 1.28$ & $1.67 \pm 0.38$ & $0.90 \pm 0.17$ & $0.36 \pm 0.09$ \\
Liver & $2.33 \pm 8.75$ & $11.17 \pm 7.77$ & $2.04 \pm 0.46$ & $1.76 \pm 0.72$ & $0.87 \pm 0.39$ \\
Bladder & $2.03 \pm 1.15$ & $1.69 \pm 1.54$ & $0.94 \pm 0.40$ & $0.68 \pm 0.10$ & $0.21 \pm 0.06$ \\
Muscle & $0.41 \pm 0.20$ & $0.32 \pm 0.18$ & $0.26 \pm 0.10$ & $0.16 \pm 0.02$ & $0.06 \pm 0.02$ \\
Skeleton & $2.07 \pm 0.84$ & $1.07 \pm 1.20$ & $0.46 \pm 0.15$ & $0.35 \pm 0.16$ & $0.13 \pm 0.03$ \\
\hline
\end{tabular}

Data are expressed as the $\% \mathrm{ID} / \mathrm{g}$ and presented as the average from 5 (occasionally 6 ) animals.

doubling time was $\sim 4$ days. In comparison with the SCC 25 cell line, tumours of this xenograft model exhibited a rather equal growth rate, providing more uniform tumour size, though the variation remined quite large.

Radiolabelling of antibodies. Labelling with ${ }^{125} \mathrm{I}$ and ${ }^{131} \mathrm{I}$ in this study resulted in overall yields of $94 \%$ for ${ }^{131} \mathrm{I}-\mathrm{cMAb} \mathrm{U} 36$ and $49 \%$ for ${ }^{125} \mathrm{I}-\mathrm{DABI}-\mathrm{cMAb} \mathrm{U} 36$. Indirect radioiodination resulted in the coupling of the 2.6 DABI prosthetic group per antibody molecule on the average. A radiochemical purity of $>95 \%$ for both conjugates was reached.

Biodistribution study. The results regarding the biodistribution of both conjugates are presented in Tables I and II. In general, the blood radioactivity concentration and the radioactivity concentration in other organs decreased over time. In contrast, tumour uptake of radioactivity increased over the first 4 days, with a subsequent decrease at day 8 for both con- 
Table III. Tumour-to-blood ratio in individual animals at 96 and $192 \mathrm{~h}$ post injection.

\begin{tabular}{lccccc}
\hline & \multicolumn{2}{c}{$96 \mathrm{~h}$} & & \multicolumn{2}{c}{$192 \mathrm{~h}$} \\
\cline { 2 - 3 } \cline { 5 - 6 } Label & DABI & CAT & & DABI & CAT \\
\hline Animal 1 & 8.56 & 5.06 & & 3.08 & 2.11 \\
Animal 2 & 6.38 & 4.63 & & 5.26 & 3.39 \\
Animal 3 & 1.98 & 1.42 & & 4.22 & 2.87 \\
Animal 4 & 4.98 & 3.38 & & 3.31 & 2.51 \\
Animal 5 & 2.86 & 2.01 & & 4.15 & 2.31 \\
P-value & \multicolumn{2}{c}{0.032} & & \multicolumn{2}{c}{0.003} \\
\hline
\end{tabular}

P-values were calculated using the paired t-test.
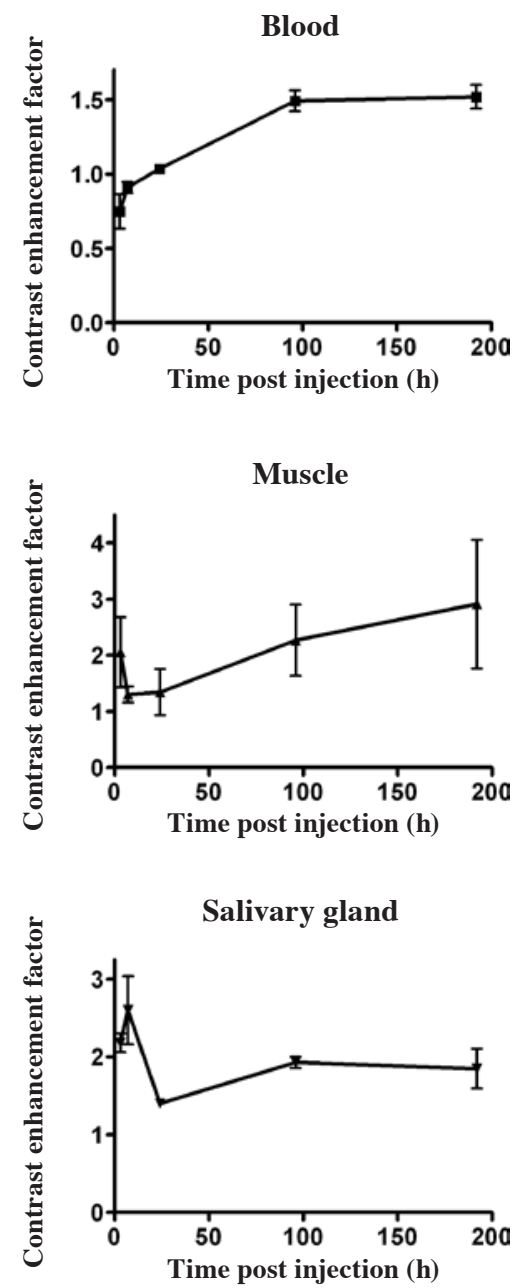

Figure 1. Contrast enhancement factors of ${ }^{125} \mathrm{I}$-DABI-cMAb U36 in comparison with ${ }^{131} \mathrm{I}-\mathrm{cMAb}$ U36. Data are presented as the mean \pm SD $(n=5-6)$.

jugates. Following injection, the accumulation of radioactivity was higher in the tumours than in the blood, other organs or tissues at time points of 24, 96 and $192 \mathrm{~h}$ for both conjugates. The only exception was the spleen, in which ${ }^{125} \mathrm{I}-\mathrm{DABI}-\mathrm{cMAb}$ U36 accumulated at $24 \mathrm{~h}$ post injection, which was higher than the accumulation in tumours at that time. This may be an indication of specific radioactivity uptake in tumours. There was
Thyroid uptake

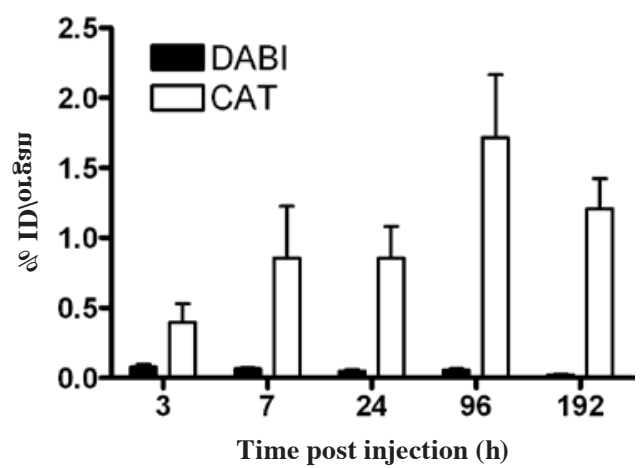

Figure 2. Comparison of thyroid uptake of ${ }^{131} \mathrm{I}-\mathrm{cMAb} \mathrm{U} 36$ and ${ }^{125} \mathrm{I}-\mathrm{DABI}-$ cMAb U36 at different times after i.v. injection. Data are presented as the mean $\pm S D(n=5-6)$

an apparent difference between the in vivo behaviour of the 2 cMAb U36-based conjugates, namely much faster blood clearance of ${ }^{125}$ I-DABI-cMAb U36 in comparison with ${ }^{131} \mathrm{I}-\mathrm{cMAb}$ U36. Elevated uptake of DABI-based conjugate in the liver and spleen was also observed.

Tumour uptake of ${ }^{131} \mathrm{I}-\mathrm{cMAb}$ U36 was higher than the uptake of ${ }^{125}$ I-DABI-cMAb U36. Since previous studies (21) demonstrated that labelling using DABI does not affect the affinity of cMAb U36 to CD44v cells in comparison with CAT labelling, the effect may have been due to the removal of ${ }^{125} \mathrm{I}$ $\mathrm{DABI}-\mathrm{cMAb} \mathrm{U} 36$ from circulation by the liver and spleen. At the same time, the comparison of individual tumours at 96 and $192 \mathrm{~h}$ post injection (Table III) demonstrated that the tumourto-blood ratio was always better for ${ }^{125}$ I-DABI-cMAb U36 in comparison with directly labelled ${ }^{131} \mathrm{I}-\mathrm{cMAb}$ U36. The use of paired labels enabled the use of the Student's t-test for paired data, which confirmed that the difference in tumour-to-blood ratio was significant.

Contrast enhancement factors, expressing the relative tumour uptake of DABI/direct labels with relative uptake in the blood, muscles and salivary glands, are presented in Fig. 1. The use of DABI was shown to provide a 1.5-fold improvement in tumour-to-blood ratio. For muscles and salivary glands, the improvement was almost 2-fold. The thyroid uptake of radioactivity was $>30$ times less using DABI in comparison with direct radioiodination (Fig. 2).

\section{Discussion}

A vital premise for the successful in vivo characterization of targeting conjugates is a suitable tumour xenograft animal model. The tumour mass affects radioactivity uptake, as is well documented in animal models (25) and in patient studies (26). Previously, the use of the SCC25 cell line with very unequal xenograft growth gave rise to extensive animal-to-animal variance in tumour size in a comparative biodistribution study (20). In the present study, the grafting rate was $100 \%$ with a doubling time of approximately 4 days. In comparison with the SCC25 cell line, this xenograft model demonstrated rather equal growth, providing more uniform tumour sizes. This makes UT-SCC7 a better candidate for experimental tumours in studies on the targeting of HNSCC. 
In this model, both ${ }^{125} \mathrm{I}-\mathrm{DABI}-\mathrm{cMAb} \mathrm{U} 36$ and ${ }^{131} \mathrm{I}-\mathrm{cMAb}$ U36 demonstrated the ability to target tumours in vivo. ${ }^{125}$ I-DABI-cMAb U36 was observed to have faster blood clearance than ${ }^{131} \mathrm{I}$-cMAb U36, most likely due to the elevated uptake of DABI-based conjugate to the liver and spleen. Previously, we observed an increased uptake of DABI-labelled cMAb in these organs in normal (21) and immunodeficient (20) mice. However, the magnitude of such uptake in the present study was appreciably lower, nor was such a profound effect had on blood kinetics. The elevated liver uptake of DABIlabelled Affibody molecules has been observed (27), though Affibody molecules are much smaller than antibodies, and linker may have a much stronger effect on their biodistribution. The reason for this phenomenon is unclear. Extensive coupling of MAG3 chelators to some antibodies including cMAb U36 was previously observed to result in the elevated uptake of ${ }^{186} \mathrm{Re}-\mathrm{cMAbs}$ in the reticuloendothelial system (the liver and spleen) immediately following the injection of the conjugates (28). Further investigation is required to clarify whether this phenomenon is label-, antibody- or animal model-dependent. The faster blood clearance of ${ }^{125}$ I-DABI-cMAb U36 made it less available and, consequently, reduced tumour uptake in comparison with ${ }^{131}$ I-cMAb U36 (Tables I and II).

The success of radioimmunodetection is determined by the contrast of the image, i.e., the difference in radioactivity concentration in the tumour compared to its surrounding tissues. The better the contrast is, the more imaging sensitivity is improved. Often, the tumour-to-blood ratio is used as a measure of contrast. An increase in the tumour-to-blood ratio, as well as an increase in the ratio of radioactivity concentration in tumours and surrounding tissues, indicates that the new labelling method provides potentially better diagnostics than conventional methods.

However, this study had its limitations. Though the UT-SCC7 xenograft provided more uniform tumour growth, the variation in tumour size remained appreciable. Fortunately, the use of the dual-label protocol enabled the comparison of the tumour-to-tissue ratio of both conjugates in the same animals. Tumour-related factors, such as size, degree of vascularisation and presence of necrotic areas, were the same for both antibodies. The comparison of individual tumours (Table III) demonstrated that the tumour-to-blood ratio was always better with ${ }^{125}$ I-DABI-cMAb U36 than with directly labelled ${ }^{131} \mathrm{I}-\mathrm{cMAb} \mathrm{U} 36$. For the quantitative characterisation of this improvement, a contrast enhancement factor was used, defined for each sample as the tumour-to-organ ratio of ${ }^{125} \mathrm{I}$ divided by the tumour-to-organ ratio of ${ }^{131}$ I for the sample, and calculated as an average for each time point.

Of note is the observed contrast with blood, muscle and thyroid. Due to the anatomical features of the tumours, radioactivity accumulation in these organs and tissues created a background that obscured tumour uptake. Contrast enhancement factors for $\mathrm{DABI} /$ direct labels in the blood, muscles and salivary glands are presented in Fig. 1, and suggest that as of 4 days post injection (approximately one physical halflife of ${ }^{124} \mathrm{I}$ ) the use of DABI provided a 1.5 -fold improved tumour-to-blood ratio. For muscles and salivary glands, this improvement was almost 2-fold. However, the results of this study do not indicate whether this improvement in contrast was due to the residualising properties of DABI, the faster blood clearance of DABI-labelled cMAb U36, or a combination of both factors.

The thyroid could pose a serious obstacle to the radioimmunodiagnostics of HNSCC using directly radioiodinated conjugates, as it avidly absorbs radiocatabolite iodide. The use of blocking with a non-radioactive iodide decreases thyroid uptake, but such blocking is seldom complete. The presence of a site with high radioactivity accumulation may give rise to artefacts during $\gamma$-scintigraphy and single photon emission computed tomography (SPECT) imaging. Our previous studies $(20,21)$ demonstrated that the use of DABI provides decreased thyroid uptake in comparison with other radioiodination methods. The present study confirmed this finding: thyroid uptake of radiodioine was $>30$ times less using DABI in comparison with direct radioiodination.

In conclusion, the UT-SCC7 cell line provides a convenient xenograft model, resulting in rapidly growing and relatively uniform tumours for studies on HNSCC targeting. In combination with the dual-label technique, nude mice with UT-SCC7 xenografts may be a convenient experimental platform for investigating the influence of various labelling methods on tumour targeting. The use of dual-label technique demonstrated that DABI as a linker for the attachment of radioiodine to cMAb U36 improves contrast between tumour and other tissues in the neck region in comparison with direct radioiodination. Enhanced contrast may improve the staging process for HNSCC using radioimmunodiagnostics.

\section{Acknowledgements}

The study was supported by the Medical Faculty University of Uppsala and the Swedish Medical Research Council, and partly supported by grants from Cancerfonden Sweden and the Erik, Karin and Gösta Selanders Foundation. The authors wish to thank Professor Guus van Dongen, who kindly donated cMAb U36 and made valuable comments on the manuscript, Professor Reidar Greenman, who kindly donated the UT-SCC7 cell line, and Dr Igor Sivaev, who prepared the potassium salt of DABI.

\section{References}

1. Parkin DM, Bray F, Ferlay J and Pisani P: Estimating the world cancer burden: Globocan 2000. Int J Cancer 94: 153-156, 2001

2. Jemal A, Tiwari RC, Murray T, et al: Cancer Statistics, 2004. CA Cancer J Clin 54: 8-29, 2004.

3. Vokes EE, Weichselbaum RR, Lippman SM and Hong WK: Head and neck cancer. N Engl J Med 328: 184-194, 1993.

4. Adjuvant chemotherapy for advanced head and neck squamous carcinoma. Final report of the Head and Neck Contracts Program. Cancer 60: 301-311, 1987.

5. Alvi A and Johnson JT: Development of distant metastasis after treatment of advanced-stage head and neck cancer. Head Neck 19: 500-505, 1997.

6. Yuen AP, Ho CM, Wei WI and Lam LK: Prognosis of recurrent laryngeal carcinoma after laryngectomy. Head Neck 17: 526-530, 1995.

7. Schuller DE, Metch B, Stein DW, Mattox D and McCracken JD: Preoperative chemotherapy in advanced resectable head and neck cancer: final report of the Southwest Oncology Group. Laryngoscope 98: 1205-1211, 1988.

8. Hart RD, Nasser JG, Trites JR, Taylor SM, Bullock M and Barnes D: Sentinel lymph node biopsy in N0 squamous cell carcinoma of the oral cavity and oropharynx. Arch Otolaryngol Head Neck Surg 131: 34-38, 131.

9. Werner JA, Dunne AA, Ramaswamy A, et al: The sentinel node concept in head and neck cancer: solution for the controversies in the N0 neck? Head Neck 26: 603-611, 2004. 
10. Adams S, Baum RP, Stuckensen T, Bitter K and Hor G: Prospective comparison of ${ }^{18} \mathrm{~F}$-FDG PET with conventional imaging modalities (CT, MRI, US) in lymph node staging of head and neck cancer. Eur J Nucl Med 25: 1255-1260, 1998.

11. Stuckensen T, Kovacs AF, Adams S and Baum RP: Staging of the neck in patients with oral cavity squamous cell carcinomas: a prospective comparison of PET, ultrasound, CT and MRI J Craniomaxillofac Surg 28: 319-324, 2000.

12. McGuirt WF, Greven K, Williams D III, Keyes JW Jr, Watson N, Cappellari JO and Geisinger KR: PET scanning in head and neck oncology: a review. Head Neck 20: 208-215, 1998.

13. De Bree R, Roos JC, Verel I, van Dongen GA and Snow GB: Radioimmunodiagnosis of lymph node metastases in head and neck cancer. Oral Dis 9: 241-248, 2003.

14. Verel I, Visser GW and van Dongen GA: The promise of immunoPET in radioimmunotherapy. J Nucl Med 46: 164-171, 2005.

15. Lundqvist $H$, Lubberink M, Tolmachev V, et al: Positron emission tomography and radioimmunotargeting - general aspects. Acta Oncol 38: 335-341, 1999.

16. Stoeckli SJ, Steinert H, Pfaltz M and Schmid S: Is there a role for positron emission tomography with ${ }^{18} \mathrm{~F}$-fluorodeoxyglucose in the initial staging of nodal negative oral and oropharyngeal squamous cell carcinoma. Head Neck 24: 345-349, 2002.

17. Verel I, Visser GW, Vosjan MJ, Finn R, Boellaard R and van Dongen GA: High-quality ${ }^{124}$ I-labelled monoclonal antibodies for use as PET scouting agents prior to ${ }^{131}$ I-radioimmunotherapy. Eur J Nucl Med Mol Imaging 31: 1645-1652, 2004.

18. Tolmachev V, Orlova A and Lundqvist H: Approaches to improve cellular retention of radiohalogen labels delivered by internalising tumour-targeting proteins and peptides. Curr Med Chem 10: 2447-2460, 2003.

19. Press OW, Shan D, Howell-Clark J, et al: Comparative metabolism and retention of iodine-125, yttrium-90 and indium-111 radioimmunoconjugates by cancer cells. Cancer Res 56: 2123-2129, 1996.

20. Cheng J, Persson M, Tolmachev V, Siavaev I, Orlova A Kairemo K and Anniko M: Targeting of a head and neck squamous cell carcinoma xenograft model using the chimeric monoclonal antibody U36 radioiodinated with a closo-dodecaboratecontaining linker. Acta Otolaryngol 124: 1078-1085, 2004.
21. Nestor M, Persson M, Cheng J, Tolmachev V, van Dongen G, Anniko M and Kairemo K: Biodistribution of the chimeric monoclonal antibody U36 radioiodinated with a closododecaborate-containing linker. Comparison with other radioiodination methods. Bioconjug Chem 14: 805-810, 2003.

22. Sivaev I BA, Nesterov VV, Antipin MY, Bregadze VI and Sjoberg S: Synthesis of schiff bases derived from the ammoniaundecahydro-closo-dodecaborate(1-) anion, $\left[\mathrm{B}_{12} \mathrm{H}_{11} \mathrm{NH}: \mathrm{CHR}\right]-$ and their reduction into monosubstituted amines $\left[\mathrm{B}_{12} \mathrm{H}_{11} \mathrm{NH}_{2} \mathrm{CH}_{2} \mathrm{R}\right]-$ : A new route to water soluble agents for BNCT. Inorg Chem 38: 5887-5893, 1999.

23. De Bree R, Roos JC, Plaizier MA, et al: Selection of monoclonal antibody E48 IgG or U36 IgG for adjuvant radioimmunotherapy in head and neck cancer patients. Br J Cancer 75: 1049-1060, 1997.

24. Orlova A, Bruskin A, Sivaev I, Sjöberg S, Lundqvist H and Tolmachev V: Radio-iodination of monoclonal antibody using potassium $\left[{ }^{125} \mathrm{I}\right]$-(4-isothiocyanatobenzylammonio)-iodo-decahydrocloso-dodecaborate (iodo-DABI). Anticancer Res 26: 1217-1223, 2006.

25. Watanabe Y, Endo K, Koizumi M, et al: Effect of tumor mass and antigenic nature on the biodistribution of labeled monoclonal antibodies in mice. Cancer Res 49: 2884-2889, 1989.

26. De Bree R, Kuik DJ, Quak JJ, et al: The impact of tumour volume and other characteristics on uptake of radiolabelled monoclonal antibodies in tumour tissue of head and neck cancer patients. Eur J Nucl Med 25: 1562-1565, 1998.

27. Tran T, Orlova A, Sivaev I, Sandström M and Tolmachev V: Comparison of benzoate- and dodecaborate-based linkers for attachment of radioiodine to HER2-targeting Affibody ligand. Int J Mol Med 19: 485-493, 2007.

28. Van Gog FB, Visser GW, Klok R, van der Schors R, Snow GB and van Dongen GA: Monoclonal antibodies labeled with rhenium-186 using the MAG3 chelate: relationship between the number of chelated groups and biodistribution characteristics. J Nucl Med 37: 352-362, 1996. 